\title{
A Study on Object Tracking Quality under Probabilistic Coverage in Sensor Networks
}

\author{
Shansi Ren Qun Li Haining Wang Xin Chen Xiaodong Zhang \\ \{sren, liqun, hnw, xinchen, zhang\}@cs.wm.edu \\ Department of Computer Science, College of William and Mary, Williamsburg, VA, USA
}

\section{Introduction}

Sensor networks are used for a wide range of object tracking applications, such as vehicle tracking in military surveillance and wild animal tracking in habitat monitoring [1]. These applications, by their nature, enforce certain tracking quality and lifetime requirements. These two requirements, however, are two conflicting optimization goals due to the stringent energy constraints of sensor nodes.

Full sensing coverage [2] is mandatory for sensor monitoring applications that require either immediate response to detected events or information of all points in the sensing field. Full sensing coverage, however, is too restricted and expensive to support long-time monitoring applications. It gives little leverage to tune object-tracking quality and battery power consumption. A relaxed sensing coverageprobabilistic coverage where any point in a sensing field is sensed with a certain probability at any timeis a more appropriate approach to balancing objecttracking quality and battery power consumption.

Probabilistic coverage scheme allows sensor nodes to periodically wake up and go back to sleep. A node in sleep mode cannot sense events; its sensing capability is resumed after it wakes up. Therefore, the sensor network provides only a fraction of the maximal coverage of all the sensors. Battery power, however, is conserved for the nodes in sleep mode. Our study aims to characterize the interplay among the sensor scheduling, tracking quality and power saving.

In this short paper, under probabilistic coverage, we present a mathematical model to analyze objecttracking quality with respect to various network conditions and sensing scheduling schemes. We define two metrics to assess object-tracking quality: the sensor detection probability (DP), and the stealth distance (SD), i.e., the distance that an object can traverse before being detected. Based on our model, we design a set of power efficient sensing protocols. We validate the correctness of our model and the effectiveness of sensing scheduling protocols through extensive simulations.

The contributions of our analytical model are three-

\begin{tabular}{|c||c|}
\hline System Parameter & Definition \\
\hline \hline$d$ & density of sensors \\
\hline$R$ & sensing radius of a sensor \\
\hline$v$ & constant velocity of a motion object \\
\hline$P$ & active ratio of sensors in $P$ \\
\hline$f$ & observation interval \\
\hline$t_{a}$ & \\
\hline
\end{tabular}

Table 1: System modeling parameters

fold. (1) This analytical model gives solid and thorough understanding about various protocols and provides insights into the pros and cons of each protocol. Even if some protocols are not amenable to easy analysis, we can approximate them and incorporate many of them into the model. (2) The analytical model helps to plan a sensor network with certain object-tracking quality requirements and power budget. It can provide accurate guidelines for optimal sensor network deployment, and can also derive the necessary speed of an object wanting to evade sensor detection. (3) Aside from determining the parameters for sensing scheduling protocols, the analytical model can direct new sensing scheduling protocol design.

\section{Object Tracking Metrics and As- sumptions}

We define the Detection Probability $(D P)$ as the expected probability that an object is detected in a given observation interval, and the Stealth Distance $(S D)$ as the average distance an object travels before it is detected. Taking energy constraints into account, we further define the Lifetime as the elapsed working time from system startup to the time when the object-tracking quality requirement cannot be met if live nodes continue sensing with their current periods, and define the Maximum Working Time as the longest possible working time of the system that satisfies the object-tracking quality requirement.

We assume that sensors are randomly and independently deployed in a field. A motion object passes through with a negligible size considering the vastness of the field. The parameters of a sensor network are summarized in Table 1. 


\section{Tracking Quality Analysis under Different Schedules}

In this section, we study random sensing schedules and synchronized sensing schedules. In a random sensing schedule, a node independently and randomly chooses the starting time of its active interval $f P$ in a sensing period $P$; while in a synchronized sensing schedule, all nodes start their active interval $f P$ simultaneously in every sensing period $P$.

\section{III.A. Random Sensing Analysis}

A random sensing schedule is a simple but usually efficient schedule due to its distributed nature. It can serve as a baseline for analysis of and comparison to other schedules.

(1) Detection probability:

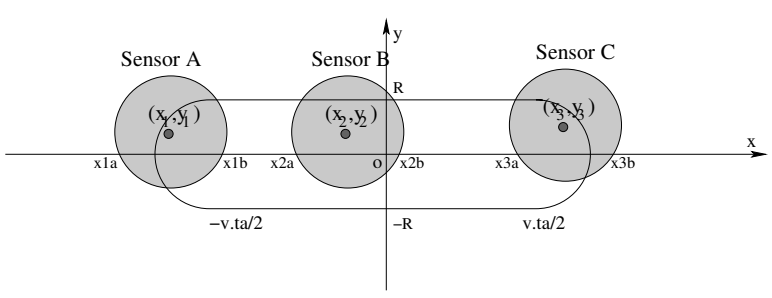

Figure 1: Three sensors in the active area of an object.

Consider a motion object that travels a distance of $\left[\frac{-v t_{a}}{2}, \frac{v t_{a}}{2}\right]$ on $x$-axis during an interval $t_{a}$. Define the active area $A A$ of this object as the oblong area in Figure 1, including the rectangle area with a length of $v t_{a}$ and a width of $2 R$, and the two half disks with radius $R$ attached to the rectangle. It can be seen that $A A=v t_{a} \cdot 2 R+\pi R^{2}$.

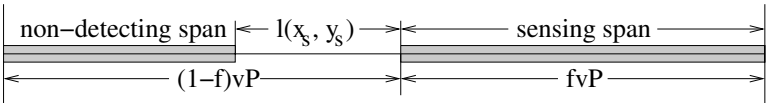

Figure 2: The distance an object moves in one period.

Let $\operatorname{Pr}\left(x_{s}, y_{s}\right)$ denote the detection probability of a sensor located at $\left(x_{s}, y_{s}\right)$ in the active area within $t_{a}$, and let $l\left(x_{s}, y_{s}\right)$ denote the intersecting length that the object trajectory intersects the node's sensing range. According to Figure 2, we have $\operatorname{Pr}\left(x_{s}, y_{s}\right)=f+\frac{t}{P}$ when $l\left(x_{s}, y_{s}\right)<(1-f) v P$, and $\operatorname{Pr}\left(x_{s}, y_{s}\right)=1$ when $l\left(x_{s}, y_{s}\right) \geq(1-f) v P$, where $t=\frac{l\left(x_{s}, y_{s}\right)}{v}$, $l\left(x_{s}, y_{s}\right)=\min \left(\frac{v t_{a}}{2}, x_{b}\right)-\max \left(\frac{-v t_{a}}{2}, x_{a}\right), x_{a}=$ $x_{s}-\sqrt{R^{2}-y_{s}^{2}}$, and $x_{b}=x_{s}+\sqrt{R^{2}-y_{s}^{2}}$.

Note that $l\left(x_{s}, y_{s}\right)=0$ and $\operatorname{Pr}\left(x_{s}, y_{s}\right)=0$ when $\left(x_{s}, y_{s}\right)$ is outside the active area. The probability that a single sensor detects this object within $t_{a}$ is $\tilde{P} r=\frac{1}{A A} \int_{-R}^{R} \mathrm{~d} y_{s} \int_{-\frac{v t_{a}}{2}-R}^{\frac{v t_{a}}{2}+R} \operatorname{Pr}\left(x_{s}, y_{s}\right) \mathrm{d} x_{s}$. Denote $\lambda=$ $d \cdot A A$. The probability that there are $k$ sensors in the active area is $\operatorname{Pr}(k)=\frac{e^{-\lambda} \cdot \lambda^{k}}{k !}, k=0,1, \ldots, \infty$, while the probability that there exists $k$ sensors in the active area and at least one of them can detect this object is $\operatorname{Pr}(d t \wedge k)=\frac{e^{-\lambda} \lambda^{k}}{k !}\left[1-(1-\tilde{P} r)^{k}\right]$. Then we get $\operatorname{Pr}($ all, rand $)=\sum_{k=1}^{\infty} \operatorname{Pr}(d t \wedge k)=$ $\sum_{k=1}^{\infty} \frac{e^{-\lambda} \cdot \lambda^{k}}{k !}\left[1-(1-\tilde{P} r)^{k}\right]=1-e^{-\lambda \tilde{P} r}$. Therefore, the expected probability that at least one sensor detects this object is $\operatorname{Pr}($ all, $r$ and $)=1-e^{-\lambda \tilde{P} r}$.

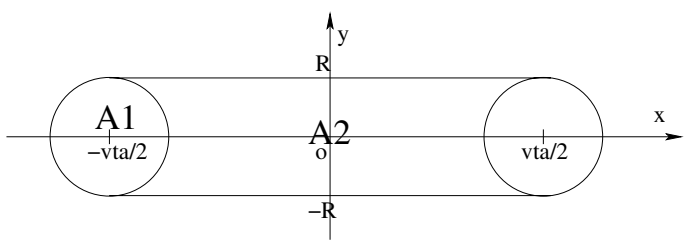

Figure 3: The integral area to calculate $D P$.

We consider an object moves with a high speed $v$ that satisfies $v t_{a}>2 R$ and $(1-f) v P>$ $2 R$. For this object, the $\tilde{P} r$ can be simplified as $\tilde{P} r=f+\frac{1}{(A A) v P} \int_{-\frac{v v_{a}}{2}-R}^{\frac{v t_{a}}{2}+R} \mathrm{~d} x_{s} \int_{-R}^{R} l\left(x_{s}, y_{s}\right) \mathrm{d} y_{s}$. Consider a sensor $s$ located at $\left(x_{s}, y_{s}\right)$. Denote $\xi_{1}=$ $\iint_{A_{1}} l\left(x_{s}, y_{s}\right) \mathrm{d} x_{s} \mathrm{~d} y_{s}$, and $\xi_{2}=\iint_{A_{2}} l\left(x_{s}, y_{s}\right) \mathrm{d} x_{s} \mathrm{~d} y_{s}$, where $A_{1}$ is the circle on the left and $A_{2}$ is the area in the middle of Figure 3. Due to the symmetry of the integral area, we have $\int_{-\frac{v t_{a}}{2}-R}^{\frac{v t_{a}}{2}+R} \mathrm{~d} x_{s} \int_{-R}^{R} l\left(x_{s}, y_{s}\right) \mathrm{d} y_{s}=$ $2 \xi_{1}+\xi_{2}$. Solve the integrals, then we have $2 \xi_{1}+\xi_{2}=$ $\pi R^{2} v t_{a}$. We can get $\tilde{P} r=f+\frac{1}{(A A) v P}\left(2 \xi_{1}+\xi_{2}\right)=$ $f+\frac{\pi R^{2} t_{a}}{\left(v t_{a} \cdot 2 R+\pi R^{2}\right) P}$, which is the expected detection probability of a single sensor for this fast moving object.

(2) Stealth distance: The $\operatorname{Pr}($ all, rand $)$ is a $c d f$ function that can be written in the form of $\operatorname{Pr}\left(t \leq t_{a}\right)$, where $t$ is the point that the object is detected for the first time, and $t_{a}$ can be viewed as a variable. Thus, $(1-c d f)\left(t_{a}\right)=\operatorname{Pr}\left(t>t_{a}\right)=e^{-\lambda \tilde{P} r}$. Because $c d f\left(t_{a}\right) \rightsquigarrow 1$ and $(1-c d f)\left(t_{a}\right) \rightsquigarrow 0$ exponentially when $t_{a} \rightsquigarrow \infty$, we have the expected detecting time $E(t)=\int_{0}^{\infty} p d f\left(t_{a}\right) \cdot t_{a} \mathrm{~d} t_{a}=\int_{0}^{\infty}(1-c d f)\left(t_{a}\right) \mathrm{d} t_{a}$. Therefore, $E\left(t_{a}\right)=\int_{0}^{\infty} e^{-\lambda \tilde{P} r} \mathrm{~d} t_{a}$. The stealth distance under a random sensing scheme is $S D=$ $\int_{0}^{\infty} e^{-\lambda \tilde{P} r} \mathrm{~d} x$, where $x=v t_{a}$ in $\tilde{P} r$.

(3) Sequential schedule and $k$-set schedule: Here we assume $2 R<(1-f) v P$, and $f P$ is constant. Let $A$ be a schedule with sensing period $k P$ and $\lambda$, where $k$ is a non-negative value. We randomly divide the nodes into $k$ equally-sized sets, and nodes in each set are randomly distributed on the field. Consider a sequential schedule $B$, where nodes in $i$ th set are active only in the duration of $[(i-1) P+n k P, i P+n k P)$ for $1 \leq i \leq k$. In the $i$ th set $S_{i}$, the detection probability 
is $\operatorname{Pr}\left(S_{i}, B\right)=1-e^{-\frac{\lambda}{k} \tilde{P} r}=1-e^{\frac{\lambda c}{k P}}$. Therefore, $\operatorname{Pr}($ all,$A)=\operatorname{Pr}($ all,$B)$.

Consider a set-based schedule, in which we randomly divide the nodes into $k$ sets $S_{1}, S_{2}, \cdots, S_{k}$. For any set $S_{i}$ with density $x_{i} \lambda$, we assign a sensing period $g_{i} P$ to it. Let $\operatorname{Pr}\left(S_{i}\right)$ denote the $D P$ for the nodes in set $S_{i}$, we know that $\operatorname{Pr}\left(S_{i}\right)=1-e^{-x_{i} \lambda \cdot \frac{c}{g_{i} P}}$. Let $\operatorname{Pr}\left(S_{i}\right)$ be the probability that no node in $S_{i}$ detects this object. If $\frac{x_{1}}{g_{1}}+\frac{x_{2}}{g_{2}}+\cdots+\frac{x_{k}}{g_{k}}=1$, then $\bar{P} r\left(S_{i}\right)=1-\operatorname{Pr}\left(S_{i}\right)=e^{-x_{i} \lambda \cdot \frac{c}{g_{i} P}}$. Thus, we have $\operatorname{Pr}($ all, rand $)=1-e^{\frac{-\lambda c}{P}}$.

\section{III.B. Synchronized Sensing Analysis}

We introduce the synchronized sensing schedule that has small standard variances on $D P$ and $S D$.

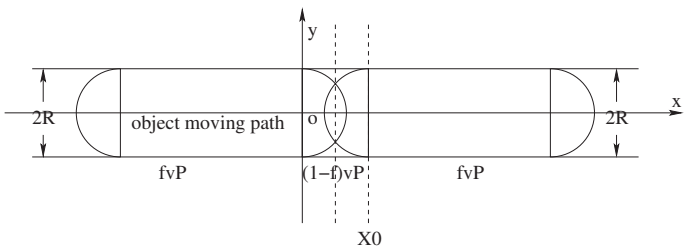

Figure 4: The active area in the synchronized schedule.

(1) Detection probability: Denote $X_{0}=(1-$ f) $v P$. According to Figure 4, during the interval $t_{a}$, the active area is $A A\left(t_{a}\right)=\pi R^{2}-I A(P)+$ $\frac{t_{a} I A(P)}{P}+2 R v f t_{a}$, where $I A(P)=X_{0} \sqrt{R^{2}-\frac{X_{0}{ }^{2}}{4}}+$ $2 R^{2} \arcsin \frac{X_{0}}{2 R}$ when $R \geq \frac{X_{0}}{2}$, and $I A(P)=\pi R^{2}$ when $R<\frac{X_{0}}{2}$. The expected detection probability that at least one sensor detects this object under the synchronized sensing scheme is $\operatorname{Pr}(a l l$, syn $)=$ $1-e^{-\lambda_{s}}$, where $\lambda_{s}=d \cdot A A\left(t_{a}\right)$.

(2) Stealth distance: Under a synchronized schedule, $S D=\frac{v P}{d \cdot(I A(P)+2 R v f P)} e^{-d\left(\pi R^{2}-I A(P)\right)}$. When $f=100 \%$, nodes remain awake all the time and never sleep, then $S D=\frac{e^{-d \pi R^{2}}}{2 d R}$.

\section{III.C. System Lifetime and Maximum Working Time}

Let $T$ be the continuous working time of a single node, and all nodes have the same $T$. Under the random schedule and the synchronized schedule, the system lifetime is $L T=\frac{T}{f}$.

Consider a simple random sensing schedule, where all nodes have identical sensing periods and only wake up once in one sensing period. In this schedule, the total energy consumption rate that meets the required tracking quality is $n f$, where $n$ is the total number of nodes. For the $n$-set schedule, consider the $i$ th node in all $n$ nodes, where $1 \leq i \leq n$, its energy consumption rate is $e r_{i}=\frac{f P}{P_{i}}$. The total energy consumption rate is $\sum_{i=1}^{n} \frac{n f P E_{i}}{E P}=n f$. In a summary, with the same $D P$ requirement, the two schedules have the same energy consumption rate, and hence, have the same maximum working time.

\section{III.D. Analytical Results and Simulation Validations}

\begin{tabular}{|c||c|c|c|c|c|c|}
\hline metric & $d \uparrow$ & $R \uparrow$ & $v \uparrow$ & $t_{a} \uparrow$ & $P \uparrow$ & $f \uparrow$ \\
\hline \hline$D P$ & $\uparrow$ & $\uparrow$ & $\uparrow$ & $\uparrow$ & $\uparrow$ & $\downarrow$ \\
\hline$S D$ & $\downarrow$ & $\downarrow$ & $\uparrow$ & & $\downarrow$ & $\uparrow$ \\
\hline
\end{tabular}

Table 2: $D P$ and $S D$ change when parameters increase.

In our simulation experiments, we generated a large square field. We plot both analytical curves and simulation results under different combinations of six parameters as shown in Figures 5, 6, and 7, respectively. Our observations are summarized as follows. (1) The simulation results match the analytical curves well, which validates the correctness of our derivations. (2) $D P$ monotonously increases, and $S D$ monotonously decreases with the increase of the parameters, as shown in Table 2. (3) The random schedule outperforms the synchronized schedule on both $D P$ and $S D$, as shown in Figure 7. (4) The stealth distance distributions have long tails: most stealth distances are short, while a few are long. The worst case of $S D$ in the random schedule is longer than that of the synchronized schedule.

\section{Design, Analysis, and Evaluation of Power Efficient Algorithms}

In this section, we design three practical sensing protocols given a fixed $f P$.

(1) Global Random Schedule (GRS): The global density $d$ is known to all sensors. Each node calculates the maximum sensing period $P_{\max }$ that satisfies the $D P$ requirement, then senses the field with $P_{\max }$.

(2) Localized Asynchronous Schedule (LAS): Every node computes its local node density $d_{l}$ individually, and uses $d_{l}$ to compute the maximum period $P_{\max }$ that meets the object-tracking quality requirement as its sensing period.

(3) Power-Aware Asynchronous Schedule (PAAS): Given the sum of the power capacities $E=\sum_{i=1}^{n} E_{i}$, we can schedule a node that has a capacity $E_{i}$ with a sensing period $\frac{E}{n E_{i}} P$.

We denote $T_{i}$ as the time when the $i$ th node runs out of its power. In GRS, all nodes have the same sensing period $P$. Denote $\delta_{i}=\frac{f P}{P}$, then $T_{1}(G R S)=$ 


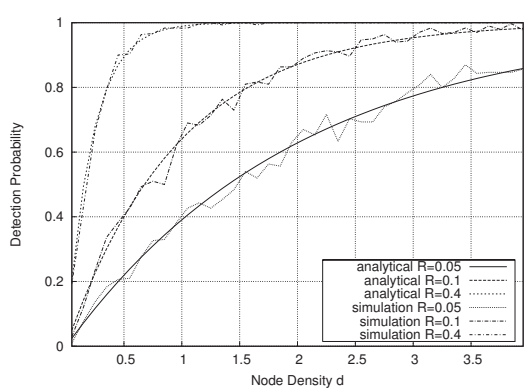

Figure 5: $D P$ changes under the random schedule when varying $d$ and $R . v=1, t_{a}=5, P=0.1$, and $f=0.5$.

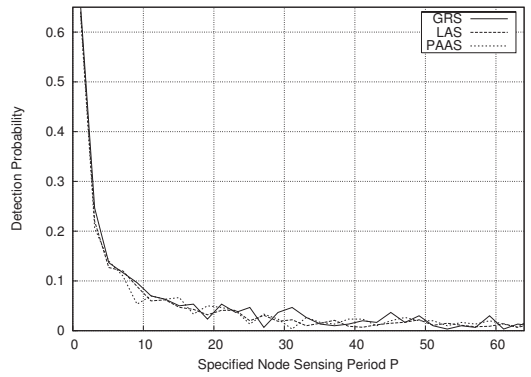

Figure 8: $D P$ changes with the increase of $P . d=0.2, R=0.5, v=$ $5, t_{a}=2, f P=0.55, r=3$.

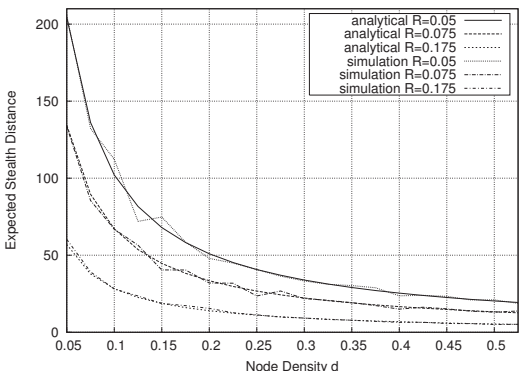

Figure 6: $S D$ changes under the random schedule when varying $d$ and $R . v=1, P=0.1, f=0.5$.

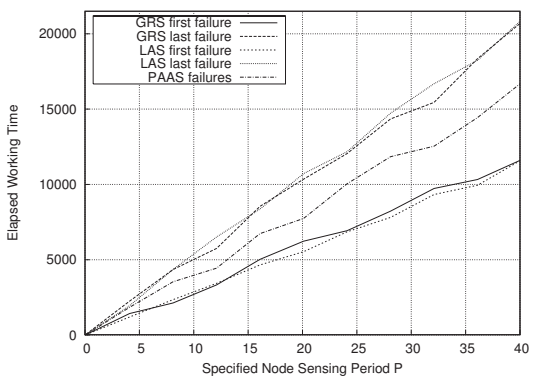

Figure 9: First/last failure time changes with the increase of $P . d=$ $2, R=0.5, v=5, t_{a}=2, f P=$ $0.1, r=3, E_{\max }=50$.

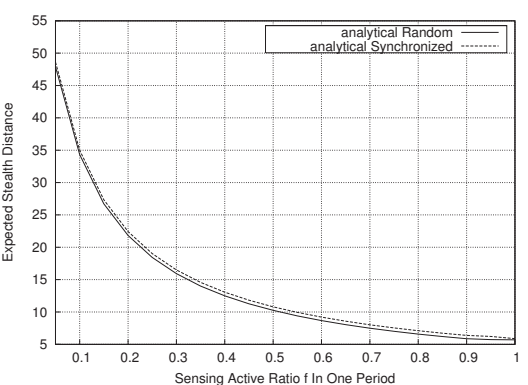

Figure 7: $S D$ changes under the random schedule and the synchronized schedule when varying $f . d=$ $0.4, R=0.2, v=2, P=2$.

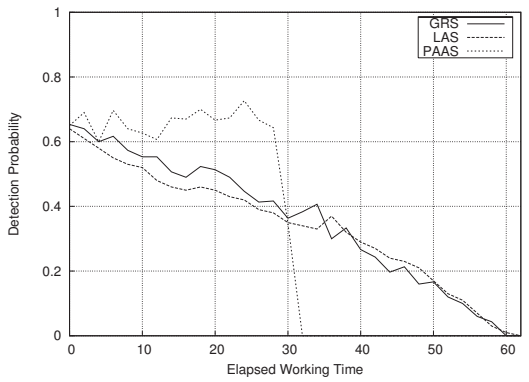

Figure 10: $D P$ decreases when time passes. $d=0.2, R=0.5, v=$ $5, t_{a}=2, P=1.1, f P=0.55, r=$ $3, E_{\text {max }}=30$. $\min \left(T_{i}\right)=\min \left(\frac{E_{i}}{f}\right)$, and $T_{1}(P A A S)=\frac{E}{f n}$, therefore $T_{1}(G R S) \leq T_{1}(P A A S)$. In other words, PAAS has a larger first failure time than GRS.

We evaluate these protocols through simulations with the same setup as that in Section III.D. The results are shown in Figures 8, 9, and 10, respectively. Every data point in the figures is obtained by averaging hundreds of trajectory runs. In Figure 9, each node's energy is $0.8 E_{\max }+e$, where $e$ follows a uniform distribution in $\left[-0.2 E_{\max }, 0.2 E_{\max }\right]$. Note that $r$ is the radio communication range of a node.

Based on our simulation results, we observe that: (1) GRS, LAS and PAAS can achieve the same DP at the beginning. (2) The first failure time and the last failure time of PAAS are the same; by contrast, GRS and LAS have smaller first failure times and larger last failure times. (3) PAAS has a longer system lifetime than GRS and LAS. (4) The DP degradation curves of GRS and LAS are exponential. The reason is that for a sensor whose energy is uniformly distributed in $\left[0, E_{\text {max }}\right]$, the $D P$ at time $t$ is $D P(t)=1-e^{-\lambda(t) \tilde{P r}}$, where $\lambda(t)=\lambda_{0}-q t, q$ is the death rate, and $\lambda_{0}$ is the initial sensor density. Thus, $D P(t)=1-e^{\lambda_{0} \tilde{P r}}$. $e^{q t \tilde{P r}}$

\section{Conclusion}

Balancing object-tracking quality and network lifetime is a challenging task in sensor networks. Under probabilistic coverage, we present an analytical model to investigate object-tracking quality with respect to various network conditions and sensing scheduling protocols. The analytical model gives us solid and thorough understanding on how to maintain objecttracking quality, and on how to provide optimal sensor deployment and conserve power consumption. Based on the model, we design three power efficient sensing protocols. The correctness of our model and the effectiveness of the proposed scheduling protocols are justified through extensive simulation experiments.

\section{References}

[1] A. Mainwaring, R. Szewczyk, D. Culler, and J. Anderson. Wireless sensor networks for habitat monitoring. In Proceedings of ACM WSNA'02, September 2002.

[2] T. Yan, T. He, and J. Stankovic. Differentiated surveillance for sensor networks. In Proceedings of ACM SenSys'03, November 2003. 